\title{
Factors Significantly Contributing to a Failed Conventional Endoscopic Stone Clearance in Patients with "Difficult" Choledecholithiasis: A Single-Center Experience
}

\author{
Emmanuel Christoforidis, ${ }^{1}$ Konstantinos Vasiliadis, ${ }^{2}$ Konstantinos Tsalis, ${ }^{1}$ \\ Dimitrios Patridas, ${ }^{1}$ Konstantinos Blouhos, ${ }^{3}$ Manousos-Georgios Pramateftakis, ${ }^{1}$ \\ Moysis Moysidis, ${ }^{1}$ and Charalampos Lazaridis ${ }^{1}$ \\ ${ }^{1}$ Fourth Surgical Department, Endoscopic Unit, "George Papanikolaou” Hospital, Aristotle University, \\ Exohi, 57010 Thessaloniki, Greece \\ ${ }^{2}$ First Department of General Surgery, Section of Hepatopancreatobiliary Surgery, "Papageorgiou" Hospital, \\ Nea Efkarpia, 56403 Thessaloniki, Greece \\ ${ }^{3}$ Department of General Surgery, General Hospital of Drama, End of Hippokratous Street, 66100 Drama, Greece
}

Correspondence should be addressed to Konstantinos Vasiliadis; keva@med.auth.gr

Received 30 May 2014; Revised 9 September 2014; Accepted 17 September 2014; Published 30 September 2014

Academic Editor: Tony C. K. Tham

Copyright (C) 2014 Emmanuel Christoforidis et al. This is an open access article distributed under the Creative Commons Attribution License, which permits unrestricted use, distribution, and reproduction in any medium, provided the original work is properly cited.

\begin{abstract}
The objective of this study is to retrospectively evaluate factors significantly contributing to a failed stone extraction (SE) in patients with difficult to extract bile duct stones (BDS). Patients and Methods. During a 10-year period 1390 patients with BDS underwent successfully endoscopic sphincterotomy. Endoscopic SE was graded as easy; relatively easy; difficult; and failed. Difficult SE was encountered in 221 patients while failed SE was encountered in 205. A retrospective analysis of the criteria governing the difficulty of endoscopic SE following the index endoscopic intervention was performed to evaluate their significance in determining failure of complete SE among patients with difficult to extract bile duct stones. Results. Age $\geq 85$ years, periampullary diverticula, multiple CBD stones $(>4)$, and diameter of CBD stones $(\geq 15 \mathrm{~mm}$ ) were all significant contributing factors to a failed SE in univariate statistical tests. In the definitive multivariate analysis age, multiple stones and diameter of stones were found to be the significant, independent contributors. Conclusion. Failed conventional endoscopic stone clearance in patients with difficult to extract BDS is more likely to occur in overage patients, in patients with multiple CBD stones $>4$, and in patients with CBD stone(s) diameter $\geq 15 \mathrm{~mm}$.
\end{abstract}

\section{Introduction}

Common bile duct (CBD) lithiasis is present in $7 \%-12 \%$ of patients with cholecystolithiasis and represents a wellestablished indication for endoscopic retrograde cholangiopancreatography (ERCP) with endoscopic sphincterotomy and basket or balloon stone extraction (SE) techniques [1]. Bile duct stones vary in size ranging from rather small (1$2 \mathrm{~mm})$ to very large $(>3 \mathrm{~cm})$ [2]. The vast majority of them $(85 \%-95 \%)$ can be removed with the use of conventional endoscopic techniques $[3,4]$. Balloon dilatation following endoscopic sphincterotomy is an easy to use alternative for difficult to extract BDS with an acceptable safety profile [5]. However, despite the refinements in endoscopic removal of $\mathrm{BDS}$, complete SE can be occasionally difficult posing an endoscopic challenge.

Extraction of BDS can be difficult for anatomic alteration and stone, duct, and patients' factors [6-9]. The size and number of BDS are major determinants of their resistance to extraction. Among other important factors, the acute distal angulation of the CBD and the shorter length of its distal CBD "arm," the altered postsurgical anatomy, and the firmness and diameter of BDS relative to the width of the distal CBD [6-9] are included. 
Failure of SE exposes the patients to a substantial risk of complications, thereby increasing morbidity and mortality [10]. In such scenario, further treatment is mandatory to avert life threatening complications [11]. Consequently, if endoscopic SE fails, patients are often referred for surgery even in the presence of substantial comorbidity [12]. The need for a definitive minimally invasive management of difficult to extract BDS forced the medical community to develop several therapeutic fragmentation modalities through shock waves both internally and externally. However, these methods are not widely available; their cost and indications are still under clinical evaluation [13]. Despite the fact that the clinical significance of factors governing the difficulty of endoscopic SE is well known, accurate identification and evaluation of these factors have attracted little investigative attention.

The objective of this study is to retrospectively evaluate factors significantly contributing to a failed SE in patients with difficult to extract BDS and to present the efficacy of conventional endoscopic management of difficult to extract BDS combined with biliary stenting when modern stone fragmentation modalities are not available.

\section{Methods}

2.1. Patients. During a 10-year period (2004 to 2014) 1390 from a total of 1448 patients with BDS underwent successful endoscopic sphincterotomy. Among these, 221 patients with difficult and 205 patients with failed SE were included in this retrospective clinical study. Patients with acute suppurative cholangitis were excluded. No oral dissolution agent was administered to the patients included in the study. Before the procedure, each patient status was classified according to the physical status classification of the American Society of Anesthesiologists (ASA) [14]. Patients with ASA score greater than 2 were excluded. Preprocedural work-up included complete blood cell count, liver function tests, serum electrolytes, and coagulation studies. All patients underwent ERCP within $24 \mathrm{~h}$ of admission. Patients were monitored during the procedure while sedation and analgesia were achieved with intravenous midazolam and fentanyl titrated to suit age and tolerance. General anesthesia was undertaken whenever this was necessary. An informed written consent was obtained from all patients. Diagnosis of BDS was based on radiologic and endoscopic findings.

2.2. Identification of Patients with Difficult Endoscopic SEGrading of Difficulty of Endoscopic SE. Although multiple factors have been postulated to govern the difficulty of endoscopic SE, it is rather difficult to objectively determine which of them represent significant and independent contributors to the failure of endoscopic clearance. In the present study, selected literature-based criteria [5-9, 15-17], added by criteria drawn from our units experience potentially contributing to the difficulty of endoscopic SE, were used for pre- and periprocedural identification of patients with presumable difficult to extract BDS. The selected criteria are listed in Table 1.
Endoscopic retrograde cholangiopancreatography (ERCP) represents the most difficult and technically demanding endoscopic procedure. Therefore, the need to objectively evaluate the difficulty of ERCP procedures has long been acknowledged. Although several US endoscopic centers have attempted to introduce ERCP technical difficulty grading scales $[18,19]$, there has been no data concerning a validated, internationally acceptable, easily understandable, reproducible grading scale, which can be applied in everyday practice as a tool to objectively evaluate the difficulty of ERCP procedures and especially the difficulty of endoscopic removal of bile duct stones. Therefore, in this study we devised an easily understood difficulty grading system according to the number of attempts at CBD stone extraction with a basket or balloon catheter taking also into consideration the patient's discomfort and the feeling of difficulty by the endoscopist. Although this grading system has not been validated, it can be easily applied in everyday practice. Besides, a similar grading scale has been used in a relevant study by Kim and coworkers [6].

Following the index endoscopic session the technical difficulty of SE was graded as follows, based on number of attempts of basket grasping or balloon sweeping: easy: completely extracted by 1 to 3 attempts; relatively easy: completely extracted by 4 to 7 attempts; difficult: completely extracted by 8 or more attempts; failed: stones incompletely removed.

\subsection{Endoscopic Procedure-Therapeutic Algorithm. All proce-} dures of ERCP, endoscopic sphincterotomy, and SE were done by the same endoscopist with a side-viewing duodenoscope, and a standard type papillotome, using the same technique with a low osmolarity, nonionic contrast media (Iopromide, Ultravist 370: Schering, Berlin, Germany). Identified patients with a presumable difficult to extract BDS followed a specific therapeutic algorithm. The first step was the catheterization of the ampulla and injection of contrast media. In case of inability to perform a cholangiogram a guide-wire was inserted into the bile duct. Provided that the latter step was successful, an endoscopic sphincterotomy was performed, followed by attempts to extract the BDS by basket-mechanical lithotripsy and/or retrieval balloon or combination of both. All sphincterotomies were complete in type, resulting in a gush of bile and the cholangiographic confirmation of air in the biliary tree. After SE, contrast media were injected in the biliary tree and the inflated balloon catheter was withdrawn along the CBD to the duodenum to confirm clearance. Clearance was then classified as difficult-successful, or failed. In the latter event, bile duct drainage was always obtained by the insertion of a plastic stent (Amsterdam type stent) or a nasobiliary catheter. Stable patients with failed SE were scheduled for a repeated, elective intervention, at which time standard techniques were again implemented. Needle knife fistulotomy (NFK) was applied in cases of inability to catheterize the ampulla and was reserved only for older patients with a dilated CBD. In our unit NFK was performed by using the NK fistulotomy technique [20]. 
TABLE 1: Selected criteria potentially contributing to the difficulty of endoscopic SE.

\begin{tabular}{|c|c|c|c|}
\hline Anatomic variations/alterations & Stone factors & Duct factors & $\begin{array}{l}\text { Patients } \\
\text { factors }\end{array}$ \\
\hline Periampullary diverticula ${ }^{a}$ & $>15 \mathrm{~mm}$ in diameter & $\begin{array}{l}\text { Acute distal CBD } \\
\text { angulation }^{\mathrm{b}}\end{array}$ & $\begin{array}{l}\text { Age } \geq 85 \\
\text { years }\end{array}$ \\
\hline Postgastrectomy Billroth type II or Roux-en-Y reconstruction & $\begin{array}{l}>4 \text { in number } \\
\text { (multiple stones) }\end{array}$ & & \\
\hline Presence of T-tube in situ + proximal lithiasis & Impacted stones & & \\
\hline Iatrogenic injury to the extrahepatic biliary tree + lithiasis & $\begin{array}{l}\text { Intrahepatic or cystic } \\
\text { duct stones }\end{array}$ & & \\
\hline
\end{tabular}

${ }^{a}$ Defined endoscopically as a depressed lesion of $5 \mathrm{~mm}$ or more with intact mucosa and with the major papilla either within it or close by [17].

${ }^{\mathrm{b}}$ Defined as the sharpest angle along the CBD from $1 \mathrm{~cm}$ below the bifurcation to $1 \mathrm{~cm}$ above the papilla [15].

2.4. Collection and Evaluation of Factors Significantly Determining Failure in Endoscopic BDS Clearance. Selected preprocedural criteria added by periprocedural parameters (including parameters identified on cholangiogram and endoscopy) that contributed to the difficulty of endoscopic SE were retrospectively collected and analyzed for their significance in determining failure in stone clearance in patients with difficult to extract BDS.

2.5. Statistical Analysis. Statistical analysis was conducted using SPSS 20.0 (SPSS Inc., Chicago, IL). The chi-squared test for independence was used in order to study univariate relationships between failure in complete SE and binary categorical variables. Factors found to be nominally significant in exploratory univariate statistical analysis were included in the binary logistic regression model to determine the significant contributors to the failure of SE. Binary logistic regression analysis was used with a stepwise procedure for model selection in order to study covariate effects on failure in endoscopic SE in a multivariable setting. The definitive results arise from the multivariate logistic regression analysis, which estimates the independent effect of each factor after adjustment for the contributions of each of the other parameters. All tests were two-sided and $P$ values less than 0.05 were considered statistically significant.

\section{Results}

All ERCPs were performed in a hospital setting. Difficult but ultimately successful SE was encountered in 221 patients while failed SE was encountered in 205 patients. The clinical characteristics and contributing factors to the difficulty of endoscopic SE in patients with difficult and failed intervention are shown in Table 2. In all patients with failed BDS clearance, endoprosthesis insertion establishing biliary drainage was a definitive measure. Patients with a failed index intervention underwent further endoscopic sessions that accomplished complete SE in either 2 (152/205, 74.1\%) or 3 sessions $(47 / 205,22.9 \%)$. All patients with a failed third endoscopic session were treated successfully surgically $(6 / 205,2.9 \%)$.

Postprocedural ERCP-related complications occurred in $34(7.98 \%)$ patients with difficult BDS (difficult and failed): acute cholangitis in 12; post-ERCP pancreatitis in 7; pulmonary complications in 7; acute cholecystitis in 5; and bleeding in 3. These complications were mild and were managed successfully conservatively with no ERCP-related deaths.

Following the index endoscopic intervention the selected contributing parameters were analyzed for the patients with difficult and failed endoscopic SE. Older age $\geq 85$ years $(P=0.028)$, periampullary diverticula $(P=0.016)$, multiple stones $>4(P=0.046)$, and diameter of CBD stones $\geq 15 \mathrm{~mm}$ $(P=0.012)$ were nominally significant contributing factors to the failure of endoscopic clearance of CBD stone(s) in exploratory univariate testing (Table 3). Presence of T-tube in situ in addition to proximal lithiasis, iatrogenic injury to the extrahepatic biliary tree in addition to CBD lithiasis, intrahepatic stones, previous gastrojejunostomy, acute distal CBD angulation, and impacted stones were not significant contributors to the failure of SE in exploratory univariate testing (Table 3 ). In the multivariate analysis it was found that age $\geq 85$ years, odds ratio $(\mathrm{OR})=1.779$, CI (1.110-2.850), $P=$ 0.017 ; multiple stones $>4$, $\mathrm{OR}=1.647$, CI (1.060-2.559), $P=$ 0.026 ; and CBD stone(s) diameter $\geq 15 \mathrm{~mm}, \mathrm{OR}=1.804, \mathrm{CI}$ (1.210-2.691), $P=0.004$, were the independent contributors to the failure of CBD stone removal (Table 4).

\section{Discussion}

Since its inception nearly 40 years ago, ERCP represents the therapeutic cornerstone for the removal of CBD stones. Following endoscopic sphincterotomy with standard extraction techniques, the vast majority of stones can be successfully removed [21]. However, in approximately $10 \%$ to $15 \%$ of patients, CBD stone removal can be difficult, predisposing to a failed endoscopic clearance [22]. Failure of SE renders the patients susceptible to the risk of cholangitis, pancreatitis, and obstructive jaundice, which add further to the significant burden of morbidity and mortality of choledocholithiasis. Therefore, assessment of contributing factors to the technical difficulty and/or failure of endoscopic SE would be extremely useful to predict a long and difficult endoscopic intervention that will require, on the one hand, special periprocedural measures, such as conscious sedation, and on the other hand, proper planning of alternative, minimally invasive therapeutic modalities, which must be carefully balanced 
TABLE 2: Clinical characteristics, preprocedural criteria, and periprocedural factors that contributed to the difficulty of endoscopic SE of patients with (a) difficult $(n=221)$ and (b) failed $(n=205)$ endoscopic BDS clearance.

\begin{tabular}{|c|c|c|}
\hline Clinical characteristics & Failed $n(\%)$ & Difficult, $n(\%)$ \\
\hline Median age in years & 78,3 & 77,1 \\
\hline \multicolumn{3}{|l|}{ Gender, $n(\%)$} \\
\hline Men & $97(47.3)$ & $100(45.25)$ \\
\hline Women & $108(52.7)$ & $121(54.75)$ \\
\hline \multicolumn{3}{|l|}{ Clinical picture, $n(\%)$} \\
\hline Jaundice/pain & $52(25.4)$ & $42(19)$ \\
\hline Abnormal blood liver test & $49(23.9)$ & $51(23.1)$ \\
\hline Acute cholangitis & $12(5.8)$ & $24(10.6)$ \\
\hline Acute pancreatitis & $9(4.4)$ & $11(64.7)$ \\
\hline Shock & $3(1.5)$ & $1(0.45)$ \\
\hline \multicolumn{3}{|l|}{ Preprocedural criteria and periprocedural factors } \\
\hline Age $\geq 85$ & $38(38)$ & $62(62)$ \\
\hline Periampullary diverticula, $n(\%)$ & $84(56.4)$ & $65(43.6)$ \\
\hline Multiple stones $>4$ & $136(44.9)$ & $167(55,1)$ \\
\hline Diameter of CBD stone $(\mathrm{s}) \geq 15 \mathrm{~mm}$ & $92(42)$ & $127(58)$ \\
\hline Presence of T-tube in situ + proximal lithiasis & $25(56.8)$ & $19(43.2)$ \\
\hline Extrahepatic biliary tree iatrogenic injury + lithiasis & $11(57.9)$ & $8(42.1)$ \\
\hline Intrahepatic stone(s) & $8(38.1)$ & $13(61.9)$ \\
\hline Previous gastrojejunostomy & $6(35.3)$ & $11(64.7)$ \\
\hline Acute distal CBD angulation & $7(46.7)$ & $8(53.3)$ \\
\hline Impacted stone(s) & $45(44.6)$ & $56(55.4)$ \\
\hline
\end{tabular}

against each other and finally with surgery, in the unfortunate event of a failed conventional endoscopic intervention. Notwithstanding, accurate determination and evaluation of factors significantly contributing to the failure of endoscopic SE have attracted little investigative attention.

Various factors have been postulated to be inversely associated with endoscopic SE, including sigmoid shaped $\mathrm{CBD}$, postgastrectomy anatomy, large number of stones, intrahepatic and cystic duct stones, and stones proximal to bile duct strictures [23]. Endoscopic retrieval of bile duct stones becomes also formidable in Mirizzi syndrome and in the presence of T-tube in situ with CBD stones proximal to the T-tube [23]. However, with the exception of stone size [24], the exact role of various parameters in the success or failure of endoscopic SE remains practically unsettled.

A valuable contribution to the exceedingly sparse literature regarding the evaluation of factors contributing to the difficulty of endoscopic SE is the study by Kim and coworkers [6]. In their prospective study it was established that older age ( $>65$ years), previous gastrojejunostomy, larger stone size $(\geq 15 \mathrm{~mm})$, impaction of stones, shorter length of the distal CBD arm ( $\leq 36 \mathrm{~mm})$, and more acute distal $\mathrm{CBD}$ angulation ( $\leq 135$ degrees) were all significant contributors to the technical difficulty for endoscopic SE. In the same study insignificant factors were found to be patients' comorbidity, previous cholecystectomy, CBD diameter $\geq$ $15 \mathrm{~mm}$, periampullary diverticula, number of CBD stones $\geq$ 5 , stone impaction, and stone $\mathrm{CBD}$ ratio $>1$. Among the aforementioned significantly contributing factors, only the shorter length of the distal CBD arm and the more acute distal CBD angulation were in the definitive multivariate analysis significant and independent contributors to the difficulty of SE [6]. However, in a similar recent prospective study by García [25], no association was found between the difficulty of endoscopic SE and the shorter length of the distal CBD arm and the more acute distal CBD angulation. Furthermore, in the study by García [25], no association was found between SE and age; previous cholecystectomy and number of stones; and stone impaction and periampullary diverticula on the contrary; stone size $\geq 15 \mathrm{~mm}$, CBD diameter $\geq 15 \mathrm{~mm}$, and mechanical lithotripsy were all found to be significant independent contributors to the difficulty of SE.

In the present study, which is probably the first evaluating certain factors that can significantly contribute to a failed endoscopic clearance in patients with difficult to extract bile duct stones, following definitive multivariate analysis, it was found that age $\geq 85$ years, multiple CBD stones $(>4)$, and the diameter of CBD stones $(\geq 15 \mathrm{~mm})$ were the significant, independent contributors to a failed endoscopic BDS clearance. In addition, the present study showed that the presence of T-tube in situ and proximal lithiasis, iatrogenic injury to the extrahepatic biliary tree in addition to CBD lithiasis, intrahepatic stones, previous gastrojejunostomy, impacted stones, and acute distal CBD angulation were not significant contributors to the failure of endoscopic clearance of $\mathrm{CBD}$ stones.

With regard to acute distal CBD angulation, it is rather logical to hypothesize that the endoscopist will encounter 
TABLE 3: Univariate analyses of contributing factors to failed endoscopic stone extraction.

\begin{tabular}{|c|c|c|c|}
\hline \multirow{3}{*}{ Age } & \multicolumn{2}{|c|}{ SE } & \multirow{2}{*}{$P$ value } \\
\hline & \multirow[t]{2}{*}{$\begin{array}{c}\text { Failed } \\
205(\%)\end{array}$} & \multirow[t]{2}{*}{$\begin{array}{c}\text { Difficult } \\
\text { successful } 221 \\
(\%)\end{array}$} & \\
\hline & & & \\
\hline$<85$ & $167(51.2)$ & $159(48.8)$ & 0.028 \\
\hline$\geq 85$ & $38(38.0)$ & $62(62.0)$ & \\
\hline \multicolumn{3}{|c|}{ Periampullary diverticula } & \multirow{3}{*}{0.016} \\
\hline No & $121(43.7)$ & $156(56.3)$ & \\
\hline Yes & $84(56.4)$ & $65(43.6)$ & \\
\hline \multicolumn{3}{|c|}{ Multiple stones } & \multirow{3}{*}{0.046} \\
\hline No & $69(56.1)$ & $54(43.9)$ & \\
\hline Yes & $136(44.9)$ & $167(55.1)$ & \\
\hline \multicolumn{3}{|c|}{ Diameter of CBD stones } & \multirow{3}{*}{0.012} \\
\hline$<15 \mathrm{~mm}$ & $113(54.6)$ & $94(45.4)$ & \\
\hline$\geq 15 \mathrm{~mm}$ & $92(42.0)$ & $127(58.0)$ & \\
\hline \multicolumn{3}{|c|}{ Presence of T-tube in situ + proximal lithiasis } & \multirow{3}{*}{ NS } \\
\hline No & $180(47.1)$ & $202(52.9)$ & \\
\hline Yes & $25(56.8)$ & $19(43.2)$ & \\
\hline \multicolumn{3}{|c|}{ Iatrogenic injury to the extrahepatic biliary tree + lithiasis } & \multirow{3}{*}{ NS } \\
\hline No & $194(47.7)$ & $213(52.3)$ & \\
\hline Yes & $11(57.9)$ & $8(42.1)$ & \\
\hline \multicolumn{3}{|c|}{ Intrahepatic stones } & \multirow{3}{*}{ NS } \\
\hline No & $197(48.6)$ & $208(51.4)$ & \\
\hline Yes & $8(38.1 \%)$ & $13(61.9)$ & \\
\hline \multicolumn{3}{|c|}{ Previous gastrojejunostomy } & \multirow{3}{*}{ NS } \\
\hline No & $199(48.7)$ & $210(51.3)$ & \\
\hline Yes & $6(35.3)$ & $11(64.7)$ & \\
\hline \multicolumn{3}{|c|}{ Acute distal CBD (common bile duct) angulation } & \multirow{3}{*}{ NS } \\
\hline No & $198(48.2)$ & $213(51.8)$ & \\
\hline Yes & $7(46.7)$ & $8(53.3)$ & \\
\hline \multicolumn{3}{|c|}{ Impacted stone } & \multirow{3}{*}{ NS } \\
\hline No & $160(49.2)$ & $165(50.8)$ & \\
\hline Yes & $45(44.6)$ & $56(55.4)$ & \\
\hline
\end{tabular}

TABLE 4: Multivariate analysis of contributing factors to successful or unsuccessful stone extraction.

\begin{tabular}{lccc}
\hline & OR & $95 \%$ CI & $P$ value \\
\hline Age & 1.779 & $1.110-2.850$ & 0.017 \\
Periampullary diverticula & 0.684 & $0.452-1.034$ & NS \\
Multiple stones & 1.647 & $1.060-2.559$ & 0.026 \\
Diameter of CBD stones $>15 \mathrm{~mm}$ & 1.804 & $1.210-2.691$ & 0.004 \\
\hline
\end{tabular}

OR: odds ratio, NS: not significant.

technical difficulties when manipulating into an abruptly deviated common duct, just above the hepatopancreatic ampulla. This difficulty owing to the tortuosity of the distal part of CBD can cause improper positioning and inadequate manipulation of stone retrieval devices. In accordance with the study by García [25] and contrary to the study by Kim et al. [6], the present study showed that acute angulation of the distal CBD proved to be an insignificantly contributing factor to a failed endoscopic SE. In our view, acute distal $\mathrm{CBD}$ angulation could play a decisive role in the difficulty of endoscopic SE in the case of coexisting dilatation of the distal CBD. In such scenario, the inflated stone extraction 
balloon cannot sufficiently rest against the wall of the dilated duct, making the retrieval of stones extremely difficult. On the contrary, in the case of an acute angulation of a nondilated distal CBD, we believe that proper manipulations, such as the "loop" handling, following the insertion of a "Jagwire" type hydrophilic guide-wire (0.035 in, Boston Scientific) proximal to the CBD angulation, can succeed in a rather straightforward SE.

These conflicting data may be explained in part by variation in operator expertise and by the fact that ERCP is a highly operator-depended intervention. However, these intrinsic procedure-related drawbacks should not discourage investigators from further clarifying and analyzing factors contributing to a failed endoscopic SE.

The present study also showed that conventional endoscopic management of difficult to extract BDS proved for the vast majority of patients safe and effective, on the condition that bile duct drainage can be obtained and patients are capable of allowing for a repeated intervention. In fact, according to the results of the present study conventional endoscopic management cleared completely the duct in the vast majority of cases with, however, the expense of multiple treatment sessions and endoprosthesis insertion. This outcome underlines the significant role of stenting when managing patients with difficult to extract BDS, which can be lifesaving [11]. Deployment of a biliary endoprosthesis is an easy and effective alternative, especially for frail, elderly, and high risk patients. Stent insertion results in immediate biliary drainage, accomplishing treatment and/or prevention of cholangitis, serves as a "bridge" to a safe, elective, repeated session or as a dissolution therapy, and prevents impaction of stones in the distal CBD thereby maintaining the biliary flow $[7,8]$.

It is worth mentioning the findings of several studies which documented that after biliary stenting for a time period ranging from three to six months, a significant proportion of large CBD stones disintegrated, decreased in size, or even disappeared [26-28]. These effects of biliary stenting, which we affirm, can serve as an adjuvant measure to finally clear initially deemed "unextractable" CBD stones.

Despite the fact that in the present study conventional endoscopic management of difficult to extract BDS proved in the vast majority of patients safe and effective, this finding should not underestimate the important role of modern stone fragmentation modalities which definitively contribute to a conclusive minimally invasive treatment, especially in cases in which various conventional therapeutic strategies fail. However, one should keep in mind that the choice, if any, of which lithotripsy technique to utilize depends on availability, expertise, and certainly economic resources.

In conclusion, failed conventional endoscopic SE in patients with difficult to extract BDS is more likely to occur in overage patients, in patients with CBD stones $>4$, and in patients with CBD stone(s) diameter $\geq 15 \mathrm{~mm}$. Endoprosthesis insertion in the event of a failed endoscopic CBD SE providing bile duct drainage offers a safety bridge to a repeated, elective, and ultimately successful minimally invasive intervention.

\section{Disclosure}

Emmanuel Christoforidis and Konstantinos Vasiliadis are equal first authors.

\section{Conflict of Interests}

The authors declare that there is no conflict of interests regarding the publication of this paper.

\section{Acknowledgment}

The authors gratefully thank Mrs. Krystallia Moshota, the former head nurse of their endoscopy unit, for her invaluableness assistance.

\section{References}

[1] M. L. Freitas, R. L. Bell, and A. J. Duffy, "Choledocholithiasis: evolving standards for diagnosis and management," World Journal of Gastroenterology, vol. 12, no. 20, pp. 3162-3167, 2006.

[2] S. Tazuma, "Epidemiology, pathogenesis, and classification of biliary stones (common bile duct and intrahepatic)," Best Practice and Research: Clinical Gastroenterology, vol. 20, no. 6, pp. 1075-1083, 2006.

[3] J. Samardzic, F. Latic, D. Kraljik et al., "Treatment of common bile duct stones-is the role of ERCP changed in era of minimally invasive surgery?" Medicinski arhiv, vol. 64, no. 3, pp. 187-188, 2010.

[4] C. Strömberg and M. Nilsson, "Nationwide study of the treatment of common bile duct stones in Sweden between 1965 and 2009," British Journal of Surgery, vol. 98, no. 12, pp. 1766-1774, 2011.

[5] G. Trikudanathan, U. Navaneethan, and M. A. Parsi, "Endoscopic management of difficult common bile duct stones," World Journal of Gastroenterology, vol. 19, no. 2, pp. 165-173, 2013.

[6] H. J. Kim, H. S. Choi, J. H. Park et al., "Factors influencing the technical difficulty of endoscopic clearance of bile duct stones," Gastrointestinal Endoscopy, vol. 66, no. 6, pp. 1154-1160, 2007.

[7] G. D. de Palma and C. Catanzano, "Stenting or surgery for treatment of irretrievable common bile duct calculi in elderly patients?" American Journal of Surgery, vol. 178, no. 5, pp. 390393, 1999.

[8] J. J. G. H. M. Bergman, E. A. J. Rauws, J. G. P. Tijssen, G. N. J. Tytgat, and K. Huibregtse, "Biliary endoprostheses in elderly patients with endoscopically irretrievable common bile duct stones: report on 117 patients," Gastrointestinal Endoscopy, vol. 42, no. 3, pp. 195-201, 1995.

[9] S. Amplatz, L. Piazzi, M. Felder et al., "Extracorporeal shock wave lithotripsy for clearance of refractory bile duct stones," Digestive and Liver Disease, vol. 39, no. 3, pp. 267-272, 2007.

[10] M. A. Parsi, T. Stevens, J. A. Dumot, and G. Zuccaro, "Endoscopic therapy of recurrent acute pancreatitis," Cleveland Clinic Journal of Medicine, vol. 76, no. 4, pp. 225-233, 2009.

[11] T. Tsujinoa, R. Sugitaa, H. Yoshidaa et al., "Risk factors for acute suppurative cholangitis caused by bile duct stones," European Journal of Gastroenterology \& Hepatology, vol. 19, pp. 585-588, 2007. 
[12] D. J. Martin, D. R. Vernon, and J. Toouli, "Surgical versus endoscopic treatment of bile duct stones," Cochrane Database of Systematic Reviews, no. 2, Article ID CD003327, 2006.

[13] S. P. Misra and M. Dwivedi, "Large-diameter balloon dilation after endoscopic sphincterotomy for removal of difficult bile duct stones," Endoscopy, vol. 40, no. 3, pp. 209-213, 2008.

[14] W. D. Owens, J. A. Felts, and E. L. Spitznagel Jr., "ASA physical status classifications: a study of consistency of ratings," Anesthesiology, vol. 49, no. 4, pp. 239-243, 1978.

[15] D. Keizman, M. I. Shalom, and F. M. Konikoff, "Recurrent symptomatic common bile duct stones after endoscopic stone extraction in elderly patients," Gastrointestinal Endoscopy, vol. 64, no. 1, pp. 60-65, 2006.

[16] D. Keizman, M. I. Shalom, and F. M. Konikoff, "An angulated common bile duct predisposes to recurrent symptomatic bile duct stones after endoscopic stone extraction," Surgical Endoscopy and Other Interventional Techniques, vol. 20, no. 10, pp. 1594-1599, 2006.

[17] D. N. Lobo, T. W. Balfour, S. Y. Iftikhar, and B. J. Rowlands, "Periampullary diverticula and pancreaticobiliary disease," British Journal of Surgery, vol. 86, no. 5, pp. 588-597, 1999.

[18] S. M. Schutz and R. M. Abbott, "Grading ERCPs by degree of difficulty: a new concept to produce more meaningful outcome data," Gastrointestinal Endoscopy, vol. 51, no. 5, pp. 535-539, 2000.

[19] R. Madhotra, P. B. Cotton, J. Vaughn et al., "Analyzing ERCP practice by a modified degree of difficulty scale: a multicentre database analysis," The American Journal of Gastroenterology, vol. 95, pp. 2480-2481, 2000.

[20] C. Mavrogiannis, C. Liatsos, A. Romanos, C. Petoumenos, A. Nakos, and G. Karvountzis, "Needle knife fistulotomy versus needle knife precut papillotomy for the treatment of common bile duct stones," Gastrointestinal Endoscopy, vol. 50, no. 3, pp. 334-339, 1999.

[21] K.-S. Yoo and G. A. Lehman, "Endoscopic management of biliary ductal stones," Gastroenterology Clinics of North America, vol. 39, no. 2, pp. 209-227, 2010.

[22] L. McHenry and G. Lehman, "Difficult bile duct stones," Current Treatment Options in Gastroenterology, vol. 9, no. 2, pp. 123-132, 2006.

[23] K. F. Binmoeller, M. Brückner, F. Thonke, and N. Soehendra, "Treatment of difficult bile duct stones using mechanical, electrohydraulic and extracorporeal shock wave lithotripsy," Endoscopy, vol. 25, no. 3, pp. 201-206, 1993.

[24] A. Lauri, R. C. Horton, B. R. Davidson, A. K. Burroughs, and J. S. Dooley, "Endoscopic extraction of bile duct stones: management related to stone size," Gut, vol. 34, no. 12, pp. 17181721, 1993.

[25] J. R. García, "Factors related to therapeutic failure in the extraction of bile duct stones for endoscopic retrograde colangiopancreatography ERCP," Revista de Gastroenterología del Perú, vol. 31, no. 4, pp. 330-334, 2011.

[26] Z. Fan, R. Hawes, C. Lawrence, X. Zhang, X. Zhang, and W. Lv, "Analysis of plastic stents in the treatment of large common bile duct stones in 45 patients," Digestive Endoscopy, vol. 23, no. 1, pp. 86-90, 2011.

[27] T. H. Lee, J.-H. Han, H. J. Kim, S. M. Park, S.-H. Park, and S.-J. Kim, "Is the addition of choleretic agents in multiple doublepigtail biliary stents effective for difficult common bile duct stones in elderly patients? A prospective, multicenter study," Gastrointestinal Endoscopy, vol. 74, no. 1, pp. 96-102, 2011.

[28] A. Horiuchi, Y. Nakayama, M. Kajiyama et al., "Biliary stenting in the management of large or multiple common bile duct stones," Gastrointestinal Endoscopy, vol. 71, no. 7, pp. 1200-1203, 2010. 


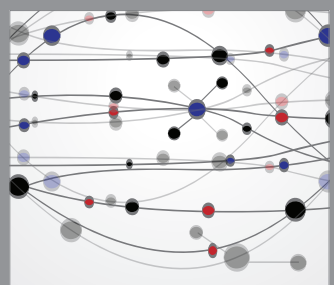

The Scientific World Journal
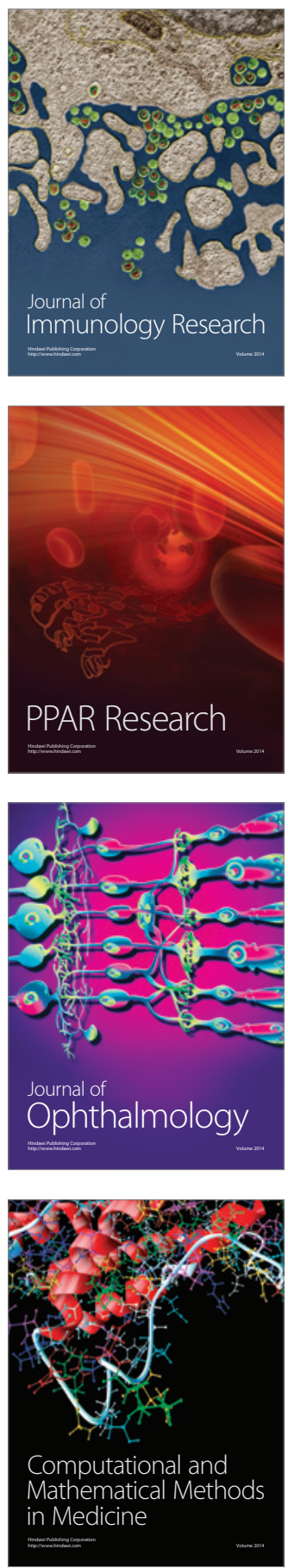

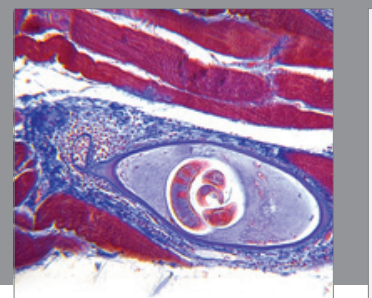

Gastroenterology

Research and Practice
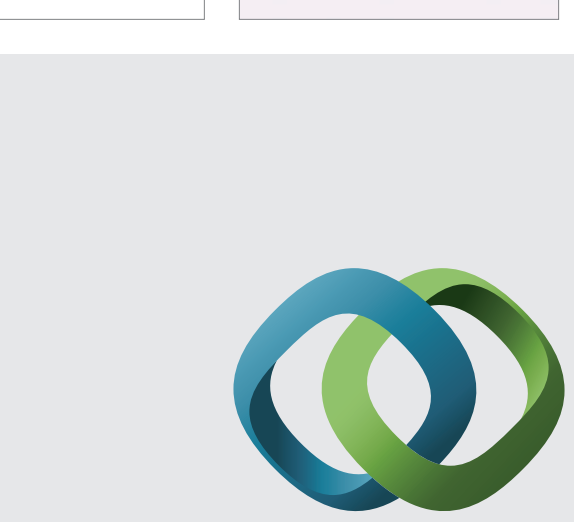

\section{Hindawi}

Submit your manuscripts at

http://www.hindawi.com
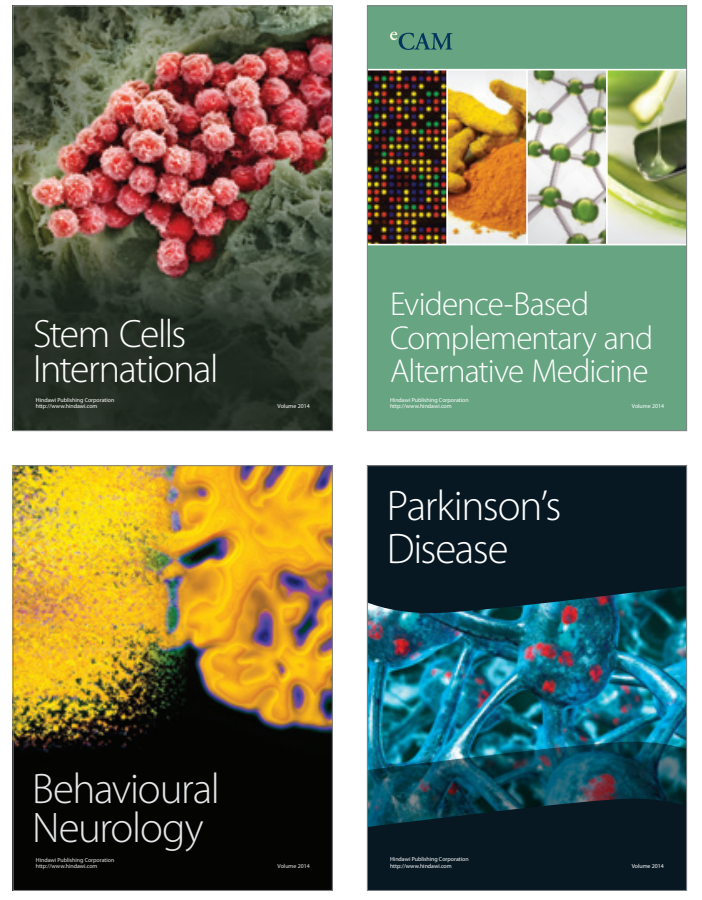
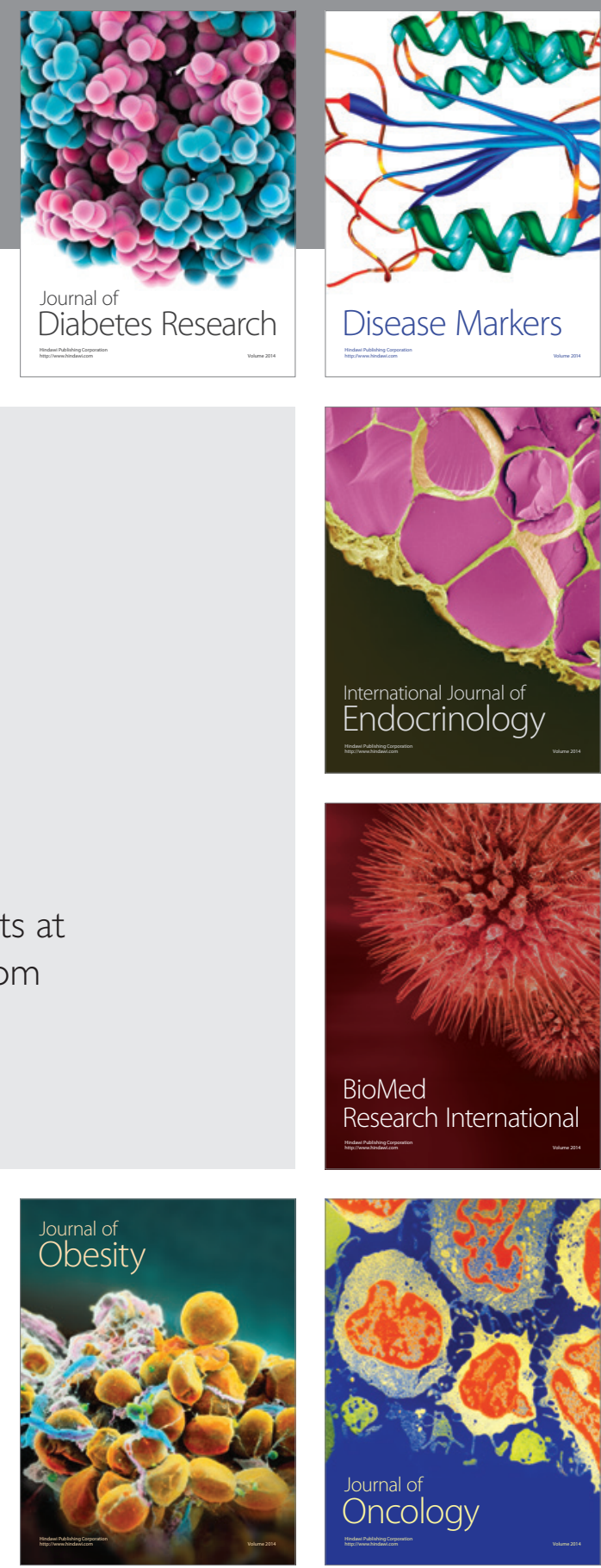

Disease Markers
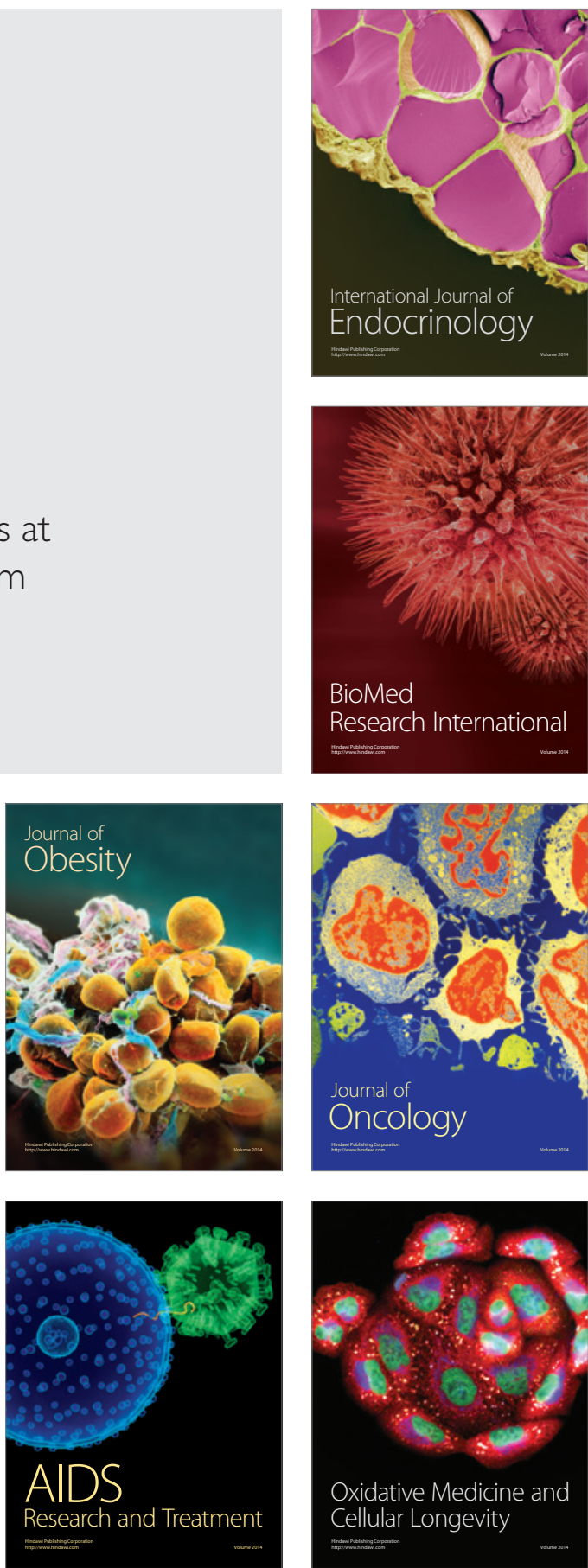